\title{
Simulation studies of a novel, charge sharing, multi-anode MCP detector
}

\section{Thomas M. Conneely*}

Photek LTD

E-mail: tom.conneely@photek.co.uk

James Milnes

Photek LTD

E-mail: james.milnes@photek.co.uk

\section{Jon Lapington}

University of Leicester

E-mail: jsl12@star.le.ac.uk

The next generation Ring Imaging Cherenkov (RICH) detectors for particle ID applications, at CERN, PANDA, and others place stringent requirements on photon detectors, with potentially high magnetic fields unaligned with the detector's optical axis, high event rates challenging detector lifetime, high density multi-anode readout and a high time resolution requirement typically less than 50 ps.

We are undertaking an $\mathrm{R} \& \mathrm{D}$ project for the TORCH collaboration, a proposed LHCb upgrade, to develop a novel multi-anode Microchannel Plate detector, with $128 \times 8$ effective pixels in a $5.3 \times 5.3 \mathrm{~cm}^{2}$ active area each with $50 \mathrm{ps}$ RMS timing resolution. The proposed design utilises charge sharing across multiple readout anodes to i) improve spatial resolution beyond the defined anode pitch, ii) provide a well-defined charge footprint for readout even in high magnetic fields and iii) reduce the impact of manufacturing issues when producing high-density multi-anode devices.

Simulations of this hybrid multi-anode detector will be presented, covering the expected charge footprint on each readout anode and the impact of utilising charge sharing between anodes to improve the detector's spatial resolution. This includes simulating the impact of the TORCH project current readout solution using the NINO amplifier/discriminator ASIC and the HPTDC, a 25 ps resolution time-to-digital converter.

Technology and Instrumentation in Particle Physics 2014,

2-6 June, 2014

Amsterdam, the Netherlands

\footnotetext{
* Speaker.
} 


\section{Introduction}

We are undertaking an R\&D project for the TORCH collaboration [1] to develop a novel multi-anode Microchannel Plate detector. TORCH (Time Of internally Reflected CHerenkov light) is a proposed ring imaging cherenkov detector to improve LHCb's particle ID capabilities, providing improved $\pi / K$ separation in the low momentum regime up to $10 \mathrm{GeV} / \mathrm{c}$. TORCH utilises a thin plane of quartz Cherenkov radiator located approximately $10 \mathrm{~m}$ from the LHCb interaction point (requiring $10-15 \mathrm{ps}$ timing resolution per particle for $3 \sigma \pi / K$ separation). The generated Cherenkov photons propagate to top/bottom of the radiator plane where they are focussed on to a bank of pixelated photon detectors using a quartz focussing block. Timing of the photon arrival at the photodetector bank, combined with the photon detector pixelation allows reconstruction of the cherenkov cone angle, the $\pi / K$ interaction point within the radiator plane and correction for chromatic dispersion of the cherenkov photons. Assuming on average 30 photons are produced per particle track, the required time resolution per photon is approximately $70 \mathrm{ps}$ [2] to achieve $10-15 \mathrm{ps}$ particle TOF resolution; of this the photon detector must account for no more than $50 \mathrm{ps}$ RMS.

Hence, to achieve the desired TOF resolution a multi-anode Microchannel Plate photomultiplier (MCP-PMT) is the chosen solution as it provides excellent single photon timing resolution ( $<30 \mathrm{ps}$ achievable [3]) and excellent spatial resolution. To achieve the required cherenkov photon angle reconstruction the MCP-PMT must achieve a spatial resolution equivalent to $128 \times 8$ pixels in a $5.3 \times 5.3 \mathrm{~cm}^{2}$ active area.

In addition to this the expected photon rate for the TORCH detector is exceptionally large at $36 \mathrm{MHz} \mathrm{cm}^{-2}$. This requirement means the MCP-PMT must have a highly parallel readout due to high occupancy and has dramatic impact on the photon detector's lifetime. Previous work $[4,5]$ has addressed the lifetime requirement using Atomic Layer Deposition to coat the MCP, reducing ageing of the detector photocathode.

To address the spatial resolution and detector occupancy requirements, a novel multi-anode readout structure has been proposed that utilises charge sharing across multiple readout anodes leading to (i) improved spatial resolution above the physical readout anode pitch, (ii) reduced number of electronic readout channels required, (iii) a well-defined charge footprint on readout anode even in high magnetic fields, and (iv) reduce likelihood of vacuum leaks from a high pixel density multi-anode device.

\section{MCP Readout Anode Design}

Traditional multi-anode MCP-PMTs use vacuum feed throughs at the rear of the detector for collecting the electron avalanche from the MCP output and coupling the signal to readout electronics. For a high pixel density device this introduces a significant probability of a vacuum leak to occuring manufacturing. Also, direct connection to readout electronics mandates that the anode be held at ground, meaning the photocathode at the front of the detector is at high voltage $(3-5 \mathrm{kV})$ complicating coupling the photocathode window to the system of interest. By using a structured multi-anode, the direct connection can be broken, allowing the anode to be at high voltage whilst reducing the chance of leakage around the feed throughs. A structured anode also allows coupling 
the MCP output charge to multiple readout anodes, with a well defined footprint, which can be used to enhance the detector's spatial resolution beyond the physical layout of the readout pads.

The charge sharing technique requires a charge measurement at each of the anode's readout pad. The photon's position $X_{\gamma}$ can then be calculated from

$$
X_{\gamma}=\sum_{i=0}^{n} x_{i} \frac{q_{i}}{Q}
$$

where $x_{i}$ is the spatial co-ordinate of a readout pad in one dimension, $q_{i}$ is the charge collected by that pad and $Q$ is the total charge collected. At the high rate, multiple events are likely to occur near simultaneously on each detector so clusters of neighbouring pads that have fired need to be identified and other pads firing rejected from the total charge sum.

The detector in development for TORCH uses a structured $8 \times 64$ structured multi-anode readout, chosen to meet detector occupancy requirements, which at $36 \mathrm{MHz} \mathrm{cm}^{-2}$ means no more than three neighbouring readout pads can be used for the charge sharing calculation. This simulation was performed to (i) optimise the structured multi-anode geometry, tuning the charge sharing footprint to maximise position resolution and meet occupancy requirements, (ii) investigate the impact of detector gain, and broad pulse height distribution of to the MCP gain process, and (iii) investigate the impact of electronic noise, timing jitter and threshold on position resolution and detector efficiency.

To meet the need for high rate, high time resolution readout with charge measurement, the NINO Time-Over-Threshold (TOT) amplifier/discriminator [6] is currently the preferred choice, as it provides a time resolution $<10 \mathrm{ps}$ at rates in excess of $10 \mathrm{MHz}$. The TOT technique also encodes the charge collected in the width of the NINO's output, however this is significantly non-linear. The NINO output pulse width and leading edge time will be digitised using the HPTDC ASIC [7], a high resolution time to digital converter with timing resolution down to $25 \mathrm{ps}$.

\section{Detector Modelling}

The first stage of the simulation was to model the response of the structured anode to the charge output of the MCP. This was performed in Ansys Maxwell, by producing a 3D model of the detector/anode structure and placing a test charge on the surface of the anode to represent the collected charge from the MCP. The capacitive coupling from the test charge at a position $(x, y)$ to each element was solved for. This allows the fraction of charge collected by each readout pad for a photon at the position $(x, y)$ to be calculated. By moving the test charge across the anode surface, a map of charge collected by each readout pad can be built up. To fill the gaps between test charge positions actually calculated, a B-spline curve was fitted and used to interpolate between data points.

This data was fed into a Monte Carlo model, which begins by modelling the MCP's current output pulse shape and gain followed by a simulation of the electronics response for photon positions generated with a uniform random distribution. For each photon, an MCP gain value was generated from a distribution fitted to a measured MCP pulse height distribution (with identical pore size). Then using the results of the Ansys Maxwell modelling, the charge collected by each 


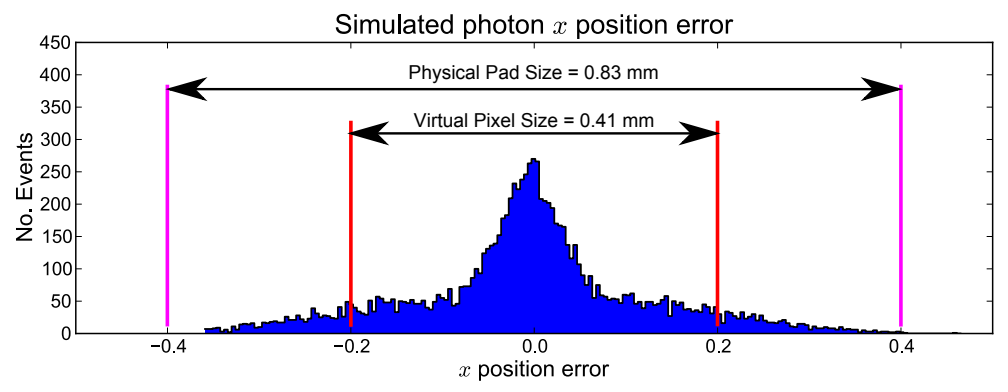

Figure 1: Simulated position error, for $10^{6}$ electron gain and $50 \mathrm{fC}$ NINO threshold.

readout pad was calculated. For each readout pad a current pulse was modelled using an analytical function

$$
\begin{gathered}
I(t)=-A e^{\frac{\left(t-t_{0}\right)^{2}}{\sigma(t)^{2}}} \\
\sigma(t)=\frac{2 \sigma_{0}}{1+e^{a\left(t-t_{0}\right)}}
\end{gathered}
$$

fitted to a real detector pulse where $A$ is the pulse amplitude (normalised so the pulse area is equal to the generated charge collected on each readout pad), $t_{0}$ is the pulse peaking time, $\sigma_{0}$ is the pulse's FWHM and $a$ detemines the asymmetry of the pulse's lead time/fall time ${ }^{1}$.

The generated detector pulse for each readout pad was passed through a model of the electronics readout. This modelled the amplifier as a low-pass filter with $1 \mathrm{~ns}$ rise time and measured the pulse's TOT, to produce an output matched against calibration data from a sample 32 channel NINO. The leading edge time/pulse width was measured and digitised into 25 ps bins to simulate the HPTDC timing format and dynamic range.

The simulated NINO's output pulse width was converted to signal charge using a previously generated charge calibration lookup table and the simulated charge for each readout pad fed into Equation 2.1 to calculate the simulated photon position. Hence, by subtracting the initial generated photon's positions from the simulated position, the spatial resolution can be studied. The detector occupancy can be studied as the charge collected by each readout anode is known.

\section{Simulation Results}

The TORCH MCP-PMT must achieve a spatial resolution equivalent to $128 \times 8$ pixels in a $5.3 \times 5.3 \mathrm{~cm}^{2}$ active area, equivalent to a pixel pitch of $0.414 \mathrm{~mm}$ in the fine direction. Hence the charge sharing resolution of the $64 \times 8$ layout, with a pitch of $0.828 \mathrm{~mm}$, must exceed this value. Figure 1 shows a typical position error distribution (generated photon position subtracted from simulated photon position), with two main contributions (i) a large central peak where more than one pad collected charge above threshold allowing a full charge sharing calculation (ii) broad tails where a single pad fired, hence no charge sharing calculation could be performed. In this case the mean number of pads above threshold per event is 1.36 , meaning there is a significant

\footnotetext{
${ }^{1}$ Values used in the simulation were $\sigma_{0}=0.220 \mathrm{~ns}, a=-3.768$ and $t_{0}=0 \mathrm{~ns}$
} 


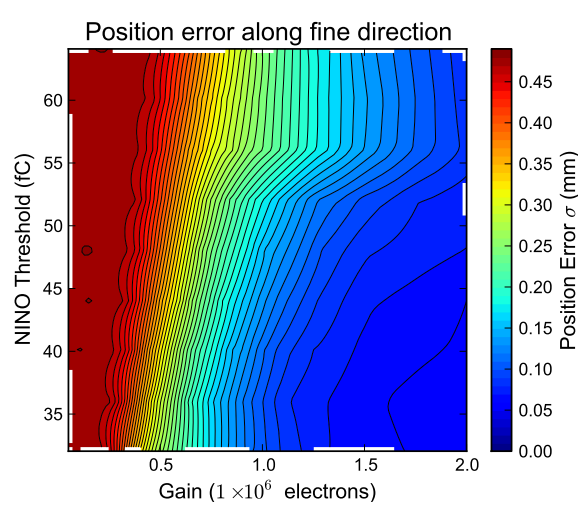

(a) Standard deviation of position error as function of NINO threshold and detector gain.

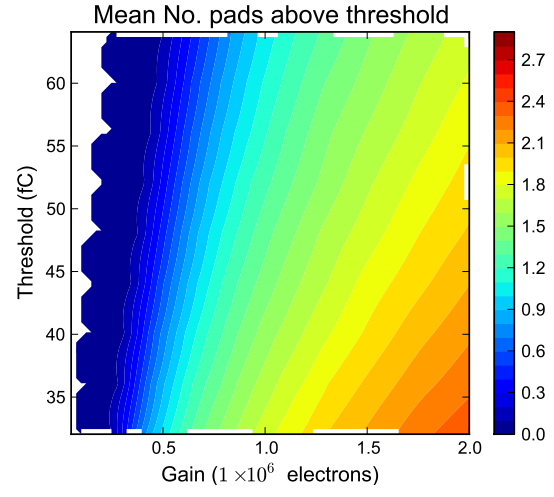

(b) Mean number of pads above threshold as function of NINO threshold and detector gain.

Figure 2

contribution from single pad events. The position resolution is taken to be the standard deviation of this distribution, which in Figure 1 is $0.19 \mathrm{~mm}$. Hence, a $0.76 \mathrm{~mm}$ equivalent pixel can be reconstructed with a $2 \sigma$ confidence level.

This position error was simulated by varying the detector gain and NINO threshold; these are the critical factors in determining SNR due to the low gains required for the high rate operation. Figure 2a shows the simulated position error standard deviation varying these two parameters, indicating for efficient charge sharing resolution a gain $>10^{6}$ is required.

To study detector occupancy, the number of readout pads with events above the NINO threshhold was used as a metric, at $36 \mathrm{MHz} \mathrm{cm}^{-2}$ this was required to be $<3$ for each event. As shown in Figure $2 b$, this criteria is comfortably met for all gains. Both of these plots were used to optimise the MCP-PMT's anode structure, as there is a tradeoff in the charge sharing measurement, especially at low gains. This occurs since the charge collected by each individual pad is reduced when charge sharing between pads is increased (i.e. the charge is spread over a larger area). Hence the SNR for each pads charge measurement is increased.

\section{Conclusion}

The results of simulating a novel type of multi-anode MCP-PMT designed for the TORCH project are presented, using charge sharing techniques to improve spatital resolution and provide additional benefits for manufacturing and operation. The simulations show the anode design meets the stringent rate requirements, and desired $0.83 \mathrm{~mm}$ spatial resolution for the TORCH project.

\section{Acknowledgements}

Photek wishes to thank members of the TORCH collaboration and the support of the European Research Council is gratefully acknowledged in the funding of the PMT development through an Advanced Grant under the Seventh Framework Programme (FP7) (ERC-2011-AdG 299175TORCH). 


\section{References}

[1] M. van Dijk et al., TORCH: a cherenkov based time-of-flight detector, Nucl. Instrum. Methods Phys. Res., Sect. A (2014).

[2] M. Charles and R. Forty, Torch: Time of flight identification with cherenkov radiation, Nucl. Instrum. Methods Phys. Res., Sect. A 639 (2011) 173 - 176.

[3] E. Ramberg, A. Ronzhin, M. Albrow, S. Los, and A. Pranko, Photodetector timing research a fermilab, Acta Physica Polonica B Proceedings Supplement 4 (2011), no. 129 - 34.

[4] T. M. Conneely, J. S. Milnes, and J. Howorth, Extended lifetime MCP-PMTs: Characterisation and lifetime measurements of ALD coated microchannel plates, in a sealed photomultiplier tube, Nucl. Instrum. Methods Phys. Res., Sect. A 732 (2013) 388 - 391.

[5] J. Milnes, T. Conneely, J. Lapington, P. Kapetanopoulos, C. Slatter, and J. Howorth, The TORCH PMT, a close packing, multi-anode, long life MCP-PMT for cherenkov applications, Nucl. Instrum. Methods Phys. Res., Sect. A (2014).

[6] F. Anghinolfi, P. Jarron, A. Martemiyanov, E. Usenko, H. Wenninger, M. Williams, and A. Zichichid, Nino: an ultra-fast and low-power front-end amplifier/discriminator asic designed for the multigap resistive plate chamber, Nuclear Instruments and Methods in Physics Research (2004) 5.

[7] M. Mota, J. Christiansen, S. Debieux, V. Ryjov, P. Moreira, and A. Marchioro, A flexible multi-channel high-resolution time-to-digital converter asic, in Nuclear Science Symposium Conference Record, 2000 IEEE, vol. 2, pp. 9/155 -9/159 vol.2, 2000. 